\title{
p-Groups are Not Determined by Their Integral Cohomology Groups
}

\author{
Journal Article
}

Author(s):

Leary, Ian

Publication date:

1995

Permanent link:

https://doi.org/10.3929/ethz-b-000422581

Rights / license:

In Copyright - Non-Commercial Use Permitted

Originally published in:

Bulletin of the London Mathematical Society 27(6), https://doi.org/10.1112/blms/27.6.585 


\title{
p-GROUPS ARE NOT DETERMINED BY THEIR INTEGRAL COHOMOLOGY GROUPS
}

\author{
IAN LEARY
}

\section{Introduction}

In this paper we prove the result of the title. More precisely, for each prime $p$ we exhibit non-isomorphic $p$-groups $G_{1}$ and $G_{2}$ such that for each $n$ the cohomology groups $H^{n}\left(G_{1}\right)$ and $H^{n}\left(G_{2}\right)$ are isomorphic. (Throughout this paper, the coefficients for cohomology are the integers unless otherwise stated.) For groups with order divisible by more than one prime, it has been known for some time that the integral cohomology ring does not determine the group. The following argument is due to Alperin and Atiyah (unpublished).

If $G$ is an extension with kernel $N$ and quotient $Q$ of coprime orders, then an easy spectral sequence argument gives the following ring isomorphism:

$$
H^{*}(G) \cong H^{*}(N)^{Q} \otimes H^{*}(Q)
$$

Thus it suffices to find a pair of groups $N$ and $Q$ of coprime orders such that $Q$ has two non-conjugate actions on $N$ having isomorphic fixed point subrings in $H^{*}(N)$. The smallest such examples have order 24 . In this case $N$ is cyclic of order three, and $Q$ may be any group of order eight having non-isomorphic subgroups $Q_{1}$ and $Q_{2}$ of order four, where the action of $Q$ on $N$ in the group $G_{i}$ is given by the faithful action of $Q / Q_{i}$. Using a different argument, Larson has been able to exhibit arbitrarily many metacyclic groups having isomorphic integral cohomology rings [4].

The only previous examples of $p$-groups having isomorphic integral cohomology groups, known to the author, are a family of (pairs of) $p$-groups for $p$ at least 5 discovered by Yagita [8], and a similar family for $p=3$, for which the author has been able to show further that the groups have isomorphic integral cohomology rings [5]. The advantages of this paper are that a wider class of examples is exhibited, including $p$-groups for $p=2$, and that very little calculation is involved. This paper was inspired by my joint work with Nobuaki Yagita, and relies on a technique suggested to me by Peter Kropholler.

\section{Methods}

Let $\tilde{G}$ be a compact Lie group whose identity component is central and isomorphic to $\mathbf{T}$, the group of complex numbers of unit modulus. If $\phi$ is a map from $\tilde{G}$ onto $\mathbf{T}$, then the pullback to $B \tilde{G}$ of the canonical bundle over $B \mathbf{T}$ is a principal T-bundle, with total space the classifying space of the group $G=\operatorname{ker}(\phi)$. Under

Received 12 March 1993; revised 1 December 1994.

1991 Mathematics Subject Classification 20J06, 20D15.

Bull. London Math. Soc. 27 (1995) 585-589 
the isomorphism (natural in $\tilde{G}) \operatorname{Hom}(\tilde{G}, \mathbf{T}) \cong H^{2}(B \tilde{G}), \phi$ corresponds to the Chern class of this bundle. The cohomology of $G$ may be computed from that of $\tilde{G}$ using the Gysin sequence for this bundle:

$$
\longrightarrow H^{n-2}(B \tilde{G}) \stackrel{\times \phi}{\longrightarrow} H^{n}(B \tilde{G}) \longrightarrow H^{n}(B G) \longrightarrow H^{n-1}(B \tilde{G}) \longrightarrow .
$$

In this long exact sequence, the map from $H^{n-2}(B \tilde{G})$ to $H^{n}(B \tilde{G})$ is multiplication by the element of $H^{2}(B \tilde{G})$ corresponding to $\phi$. The idea of using this technique to study the cohomology of a finite group was suggested by P. H. Kropholler and J. Huebschmann [2, 3].

Notation. From here onwards, $\tilde{G}$ will stand for a Lie group as above (that is, with central identity component isomorphic to $T$ ), such that the group of components $\tilde{G} / \mathbf{T}$ is a finite $p$-group for some prime $p$.

Our examples will be pairs of finite subgroups of such a group $\tilde{G}$ corresponding to similar elements in $\operatorname{Hom}(\tilde{G}, \mathbf{T})$. Before stating our condition for the cohomology groups of two such groups to be isomorphic, in Lemma 4, we require three propositions, one concerning the cohomology of groups such as $\tilde{G}$, one concerning the cohomology of their finite subgroups, and another which will allow us to unfilter a result obtained from a spectral sequence.

Proposition 1. If $\tilde{G}$ is a compact Lie group as above, then for $n$ odd $H^{n}(B \tilde{G})$ is a finite p-group, and for $n$ even $H^{n}(B \tilde{G})$ is the direct sum of a finite p-group and an infinite cyclic group. If $\theta$ is an element of infinite order in $H^{2}(B \tilde{G})$, then multiplication by $\theta$ sends any element of infinite order in $H^{n}(B \tilde{G})$ to an element of infinite order in $H^{n+2}(B \tilde{G})$. The exponent of torsion in $H^{*}(B \tilde{G})$ is bounded by the order of the group of components of $\tilde{G}$.

Proof. Let $T$ be the identity component of $\tilde{G}$. In the Serre spectral sequence for the fibration

$$
B T \longrightarrow B \tilde{G} \longrightarrow B(\tilde{G} / T),
$$

the $E_{2}$-page is isomorphic to the tensor product of the cohomology of $T$ and that of $\tilde{G} / T$. Thus $E_{2}^{i, j}$ is a finite $p$-group except for $E_{2}^{0,2 j}$ which is infinite cyclic, and hence the same is true for $E_{n}^{i, j}$ for all $n$. The $E_{\infty}$-page now yields a finite filtration of $H^{n}(B \tilde{G})$ in which every subquotient is a finite $p$-group, except the top one, which is infinite cyclic if $n$ is even. An element of infinite order in $H^{2 m}(B \tilde{G})$ yields a non-zero element of $E_{\infty}^{0,2 m}$, and the product of such an element with a non-zero element of $E_{\infty}^{0,2}$ will be a non-zero element of $E_{\infty}^{0,2 m+2}$. Finally, multiplication by the order of the group of components factors as the composite of the restriction map from $\tilde{G}$ to $T$ followed by the transfer back to $\tilde{G}$, and $H^{*}(B T)$ is torsion free, so this map must annihilate torsion.

REMARK. If $G$ is the kernel of a map from $\tilde{G}$ to $\mathbf{T}$, then it is easy to see that $G$ is finite if and only if the map is onto, which occurs if and only if the map has infinite order when viewed as an element of $\operatorname{Hom}(\tilde{G}, \mathbf{T})$.

Proposition 2. If $G$ is the kernel of a map from $\tilde{G}$ onto $\mathbf{T}$ and $p^{l}$ annihilates the torsion in $H^{*}(B \tilde{G})$, then $p^{2 l} H^{2 n+1}(B G)$ is trivial, and $p^{2 l} H^{2 n}(B G)$ is cyclic. 
Proof. Consideration of the Gysin sequence for $B G$ as a T-bundle over $B \tilde{G}$ shows that $H^{n}(B G)$ is expressible as an extension with kernel a quotient of $H^{n}(B \tilde{G})$ and quotient a subgroup of $H^{n-1}(B \tilde{G})$. Each of these groups must be finite, because $H^{n}(B G)$ is finite. By Proposition 1, a finite subgroup of $H^{n-1}(B \tilde{G})$ has exponent dividing $p^{l}$, and a finite quotient group of $H^{n}(B \tilde{G})$ has exponent $p^{l}$, except that when $n$ is even it may have one cyclic summand of higher order. The result now follows.

Proposition 3. If $A$ is a finite abelian p-group such that $p^{m} A$ is cyclic, then the isomorphism type of $A$ is determined by the order of $A$ and the orders of $\mathbf{Z}_{l p^{k}} \otimes A$ for $k \leqslant m$.

Proof. Express $A$ as a sum of cyclic groups, and let $n(k)$ stand for the number of cyclic summands in this expression of order at least $p^{k}$. By assumption, $n(m+1)$ is at most one, and we may recover the $n(k)$ for $k \leqslant m$ from the following equation:

$$
p^{n(k)}=\left|\mathbf{Z}_{/ p^{k}} \otimes A\right| /\left|\mathbf{Z}_{/ p^{k-1}} \otimes A\right| .
$$

This determines all of the cyclic summands of $A$ except the largest one, which is obtainable now from $|A|$.

LEMMA 4. Let $\tilde{G}$ be a Lie group as above, and let $\theta, \psi$ be elements of $\operatorname{Hom}(\tilde{G}, \mathbf{T})$ such that $\theta$ has infinite order and $\psi$ has finite order. Now let $\phi_{1}=p^{m} \theta+\psi$ and $\phi_{2}=p^{m} \theta+q \psi$, where $q$ is an integer coprime to $p$, and let $G_{i}$ be the kernel of $\phi_{i}$. Then for sufficiently large $m$, the groups $H^{n}\left(B G_{1}\right)$ and $H^{n}\left(B G_{2}\right)$ are isomorphic for all $n$.

Proof. In fact, if $p^{l}$ annihilates the torsion in $H^{*}(B \tilde{G})$, we may take any $m$ greater than or equal to $2 l$, and for the rest of the proof we fix some $m$ having this property. We shall consider the Gysin sequences for $B G_{1}$ and $B G_{2}$ as T-bundles over $B \tilde{G}$ for various choices of coefficients. First we use the Gysin sequence with integer coefficients to show that $H^{n}\left(B G_{1}\right)$ and $H^{n}\left(B G_{2}\right)$ have the same order. Let $T^{n}$ stand for the torsion subgroup of $H^{n}(B \tilde{G})$, and let $F^{n}$ be a complement to $T^{n}$, so that $F^{n}$ is either infinite cyclic or trivial. If we define an automorphism $f$ of $H^{n}(B \tilde{G})$ as the sum of the identity map on $F^{n}$ and multiplication by $q$ on $T^{n}$, then because $p^{m}$ annihilates $T^{n}$, the following diagram commutes.

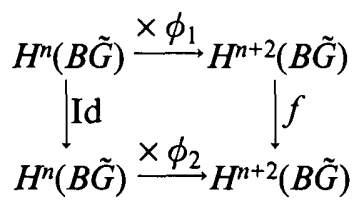

It follows that the kernels of $\times \phi_{1}$ and $\times \phi_{2}$ have the same order, and similarly for the cokernels, and hence that $H^{n}\left(B G_{1}\right)$ and $H^{n}\left(B G_{2}\right)$ have the same order.

Next consider the Gysin sequences with $\mathbf{Z}_{/ p^{k}}$ coefficients for $k \leqslant m$. For these sequences the maps from $H^{n}(B \tilde{G})$ to $H^{n+2}(B \tilde{G})$ differ only by a unit multiple, and so have identical kernels and cokernels. It follows that $H^{n}\left(B G_{1} ; \mathbf{Z}_{/ p^{k}}\right)$ and $H^{n}\left(B G_{2} ; \mathbf{Z}_{/ p^{k}}\right)$ have the same order for each $k \leqslant m$. Now the universal coefficient theorem

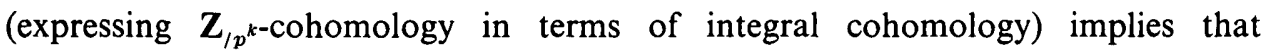
$\mathbf{Z}_{/ p^{k}} \otimes H^{n}\left(B G_{1}\right)$ and $\mathbf{Z}_{/ p^{k}} \otimes H^{n}\left(B G_{2}\right)$ have the same order for all $n$ and for all $k \leqslant m$. We are now able to apply Proposition 3 (since $p^{m} H^{n}\left(B G_{i}\right)$ is cyclic by Proposition 2), and deduce that for all $n, H^{n}\left(B G_{1}\right)$ and $H^{n}\left(B G_{2}\right)$ are isomorphic. 


\section{Examples}

It remains to construct, for each prime $p$, pairs of non-isomorphic $p$-groups satisfying the conditions of Lemma 4. First we consider odd primes. Fix an odd prime $p$, and $n$ a divisor of $p-1$. Now for any $m \geqslant 0$ and any integer $q$ coprime to $p$, define a group $G=G(m, q)$ by the presentation

$$
\begin{aligned}
G=\langle A, B| G^{\prime \prime}=1, A^{p^{m+2}=} & B^{p}=1,\left[A, G^{\prime}\right]=1, \\
& {\left.\left[A^{p}, G\right]=1,[B, \ldots[B,[B, A]] \ldots]=A^{p^{m+1}}\right\rangle, }
\end{aligned}
$$

where the letter $B$ occurs $n$ times in the repeated commutator and $G^{\prime}$ stands for the derived subgroup of $G$. The subgroup of $G$ generated by $A$ and the derived subgroup is abelian and isomorphic to $C_{p^{m+2}} \oplus\left(C_{p}\right)^{n-1}$, and $G$ is the split extension with kernel this group and quotient of order $p$ generated by $B$. It is easy to see that $G(m, q)$ and $G(m, p+q)$ are isomorphic via a map sending $A$ to $A$ and $B$ to $B$, so that the isomorphism type of $G$ depends only on $m$ and the image of $q$ in $\mathbf{F}_{p}^{\times}$. Any elements $A^{\prime}$ and $B^{\prime}$ generating $G$ such that $A^{\prime}$ commutes with $G^{\prime}$ and $B^{\prime}$ has order $p$ must satisfy a similar presentation, except that the new $q$ may be the old one multiplied by the $n$th power of any integer coprime to $p$. It follows that for fixed $m$ there are $n$ isomorphism types of such groups. For $n=2$ these are the groups already considered by the author and Yagita $[5,8]$.

For all $m$ and $q$, these groups are normal subgroups of the Lie group $\tilde{G}$ with presentation

$$
\begin{aligned}
\tilde{G}=\langle X, Y, \mathbf{T}| \tilde{G}^{\prime \prime}=1, X^{p}= & Y^{p}=1,[\mathbf{T}, \tilde{G}]=1, \\
& {\left.\left[X, \tilde{G}^{\prime}\right]=1,[Y, \ldots[Y,[Y, X]] \ldots]=\exp (2 \pi i / p)\right\rangle, }
\end{aligned}
$$

and the corresponding homomorphisms from $\tilde{G}$ to $\mathrm{T}$ are related as required by Lemma 4. More precisely, let $\theta$ be the homomorphism from $\tilde{G}$ to $\mathbf{T}$ that sends $X$ to 1 and $Y$ to 1 , and restricts to $T$ as $z \mapsto z^{p}$. Now let $\psi$ be the homomorphism from $\tilde{G}$ to $\mathbf{T}$ that sends $\mathbf{T}$ to $1, Y$ to 1 and $X$ to $\exp (-2 \pi i / p)$. For $q$ coprime to $p$, the kernel of $p^{m} \theta+q \psi$ is generated by $X \exp \left(2 \pi i q / p^{m+2}\right)$ and $Y$, and is isomorphic to $G\left(m, q^{\prime}\right)$, where $q q^{\prime} \equiv 1$ modulo $p$.

Now consider the 2-groups $H(m, q)$ where $m \geqslant 0$ and $q$ is 1 or 3 , with the following presentations:

$$
H=\left\langle A, B \mid A^{2^{m+4}}=B^{4}=1,\left[A^{4}, H\right]=1,\left[A, H^{\prime}\right]=1,[B,[B, A]]=A^{q 2^{m+2}}\right\rangle .
$$

The groups $H(m, 1)$ and $H(m, 3)$ are not isomorphic, by reasoning similar to that given above. Let $\tilde{H}$ be the Lie group with presentation

$$
\tilde{H}=\left\langle X, Y, \mathbf{T} \mid X^{4}=Y^{4}=1,[\mathbf{T}, \tilde{H}]=1,\left[X, \tilde{H}^{\prime}\right]=1,[Y,[Y, X]]=i\right\rangle .
$$

Let $\theta$ from $\tilde{H}$ to $\mathbf{T}$ be defined by $\theta(X)=1, \theta(Y)=1, \theta(z)=z^{4}$, and let $\psi$ from $\tilde{H}$ to $\mathrm{T}$ be defined by $\psi(X)=-i, \psi(Y)=1, \psi(z)=1$. If $q$ is 1 or 3 , then the kernel of the map $2^{m} \theta+q \psi$ is generated by $\exp \left(\pi i q / 2^{m+3}\right) X$ and $Y$, and is isomorphic to $H(m, q)$.

COROLlary 5. For each prime p, there are distinct p-groups with isomorphic integral cohomology groups.

Proof. Apply Lemma 4 to the examples given above. 
REMARKS. In contrast to Corollary 5, a result due to Stallings [6] and Stammbach [7] implies that a map $f: G_{1} \rightarrow G_{2}$ between $p$-groups must be an isomorphism if it induces an isomorphism between $H^{1}\left(B G_{2} ; \mathbf{Z} / p\right)$ and $H^{1}\left(B G_{1} ; \mathbf{Z} / p\right)$, and an injection from $H^{2}\left(B G_{2} ; \mathbf{Z} / p\right)$ to $H^{2}\left(B G_{1} ; Z / p\right)$.

The author does not know of a pair of finite groups of different orders having isomorphic integral cohomology groups. In [1], Carlson introduced an integer invariant che $(G)$ for a finite group $G$. The invariant can be defined in terms of the additive structure of $H^{*}(B G)$, and is a multiple of the order of $G$, but this multiple depends on $G$ [1].

\section{References}

1. J. F. Carlson, 'Exponents of modules and maps', Invent. Math. 95 (1989) 13-24.

2. J. HuebschmanN, 'Perturbation theory and free resolutions for nilpotent groups of class 2 ', J. Algebra 126 (1989) 348-399.

3. J. HuEbSCHMANN, 'Cohomology of nilpotent groups of class 2', J. Algebra 126 (1989) 400-450.

4. D. S. LARSON, 'The integral cohomology rings of split metacyclic groups', Unpublished report, University of Minnesota (1987).

5. I. J. LEARY, '3-groups are not determined by their integral cohomology rings', J. Pure Appl. Algebra, to appear.

6. J. Stallings, 'Homology and central series of groups', J. Algebra 2 (1965) 170-181.

7. U. StammвaCH, 'Angewandungen der Homologietheorie der Gruppen auf Zentralreihen und auf Invarianten von Präsentierungen', Math. Z. 94 (1966) 155-177.

8. N. Y AGITA, 'Cohomology for groups of $\operatorname{rank}_{p}(G)=2$ and Brown-Peterson cohomology', J. Math. Soc. Japan 45 (1993) 627-644.

Departement Mathematik

ETH Zentrum

8092 Zürich

Switzerland
Current address

Centre de Recerca Matemàtica

Institut d'Estudis Catalans

E 08193 Bellaterra

Spain 